\title{
Impact of MAC Layer on AODV and LAR Routing Protocols in MANETs
}

\author{
A.Venkataramana \\ Research Scholar, \\ Dept. of CS\&SE \\ Andhra University, \\ Visakhapatnam, \\ Andhra Pradesh, India
}

\author{
S.Pallam Shetty, Ph.D \\ Professor \\ Dept. of CS\&SE \\ Andhra University \\ Visakhapatnam \\ Andhra Pradesh, India
}

\begin{abstract}
A Mobile Ad hoc Network is a type of ad hoc network. It can change its location configuration dynamically. It is an infrastructure-less network. Due to the complex nature of MANETS, their development processes face several challenges. One of such key challenge is routing. Several routing protocols have already been proposed for MANETs. This paper examines the impact of MAC protocols 802.11 and CSMA on the two on demand routing protocols AODV and LAR in MANETs with varying Node density in the network. Number of simulation scenarios was carried out by using Glomosim-2.03. The simulation metrics used are Throughput, Delay and PDR. Simulation experiments found that both 802.11 and CSMA is suitable for AODV where as only 802.11 is suitable for LAR.
\end{abstract}

\section{General Terms}

Wireless networks, Mobile ad hoc Networks, MAC Protocols, Routing Protocols

\section{Key words \\ AODV, LAR, CSMA, GloMoSim, PDR.}

\section{INTRODUCTION}

Mobile Ad hoc Networks is a collection of autonomous mobile nodes that are dynamically communicating without any centralized administration. It is a self-creating, self-arrange and self-regulating network. In this network each node plays dual role i.e., node as well as router. In Mobile ad hoc network, nodes are having high mobility; because of this mobility routing is an important issue in Ad hoc network. An efficient routing protocol, which provides QoS by minimizing delay and power consumption while maximizing throughput and utilization of resources, remains a challenge issue for the adhoc network. MAC layer plays key role in Routing, so the selection of routing protocols and impact of MAC layer on routing protocols is one of the research areas in MANETs.

Medium Access Control (MAC), is part Data Link Layer (layerl-2 of the OSI reference model), this protocol is used for provide channel access mechanism when multiple users are trying to access the single channel [11]. Some of the MAC protocols are 802.11 DCF, CSMA and MACA. This paper investigates the impact of AODV and LAR routing protocols for MAC layer protocols CSAM and 802.11. The performance of the protocols will be best when run over IEEE 802.11, comparing with CSMA, due to its channel acquisition characteristics [3]. However, for some parameters CSMA also show good performance. The aim of this paper is to determine whether the selection of MAC protocol is a factor when comparing routing protocols, this paper investigate the behavior of AODV and LAR routing Protocols when run over varying MAC protocols.

The rest of the paper is organized as follows. Section II, present the overview of routing protocols. In Section III, describes the MAC layer protocols IEEE 802.11 and CSMA. The simulation environment Results are showed in Section IV \& V respectively. The paper is concluded in Section VI.

\section{ROUTING PROTOCOLS}

Routing is nothing but finding the shortest and optimal path from source to destination in any network. At least one intermediate node within the internetwork is encountered during the transfer of information [11]. Routing Protocols plays crucial role in MANETS. Routing protocol for ad-hoc network can be categorized in to three categories. The three classifications of routing algorithms are Reactive, Proactive and Hybrid routing protocols. Pro-active routing is also called Table driven routing where as re-active routing is called Ondemand \& dynamic routing. Reactive routing protocols are popular in MANETs because they are more scalable and less overhead on the network. LSR and DSDV are the pro-active routing protocols. AODV and LAR are the popular Re-active routing protocols [1]. In the reactive routing protocol, the routes are created only when a node wants to send data to another node in the network (i.e., on demand). There are no predefined routes. Its main advantage is the reduced overhead on the network because there is no need to exchange information about the network topology. On the other hand, it increases the time to find the route, and the source must reinitiate a new route request when the old has failed. This paper explains two re-active routing protocols AODV and LAR in the following subsections.

\subsection{Ad hoc on-demand Routing}

The AODV is a reactive routing protocol. i.e., a route is established only when it is required by a source node. It reacts to the changes. It maintains only the active routes in the tables for a pre-specified expiration time. These routes are found and are expected to be available at a given instant. It also performs unicast routing. It employs destination sequence numbers to identify the most recent path [2]. AODV adopts the destination sequence number technique. AODV uses three types of packets for establishing and maintain routes. These are Route Request (RREQ), Route Reply (RREP) and Route Error (RRER)

RREQ - Source node initiates a route discovery process if no route is available in the routing table. It broadcasts the demand through the RREQ packets. Each RREQ is has an ID and the address of the source and destination in its header. It expects return acknowledgement from destination. Each RREQ starts 
with a small Time to Live (TTL) value. If the destination is not found during the TTL, The TTL is increased in subsequent RREQ packets.

RREP - A route reply packet is created and forwarded back to the source only if the destination sequence number is equal to or greater than the one specified in RREQ. It guarantees updating of routing catch information. Route table keep the all entries in a cache, which contains the received RREQs. Only the RREQs of highest sequence number are accepted and all previous ones are discarded [6].

RERR - Route Error packet are generated by intermediate nodes and forwarded back to source to inform the route errors. AODV uses only symmetric links. Each intermediate node will alert the source by sending RERR packets [12].

\subsection{Location aided routing}

Location-aided routing (LAR) is a mechanism which attempts to reduce the control message overhead of Ad-hoc on-demand distance vector (AODV) routing protocol by flooding only the portion of the network that is likely to contain the route to destination. LAR takes advantage of Global Positioning System (GPS) coordinates to identify a possible location of the destination node [7]. Based on this information, LAR defines a portion of the network which will be subject to the limited flooding, thus reducing the total number of the control packet traveling through the network during the route discovery process. The proposed approach is termed Location-Aided Routing (LAR), as it makes use of location information to reduce routing overhead. Location information used in the LAR protocol may be provided by the Global Positioning System (GPS). With the availability of GPS, it is possible for a mobile host to know its physical location [5]. In reality, position information provided by GPS includes some amount of error, which is the difference between GPS-calculated coordinates and the real coordinates.

\section{MAC LAYER PROTOCOLS}

In the Mobile Ad-hoc Networks (MANETs), MAC and routing are two major layers that need careful consideration. MAC belongs to second layer in the OSI reference model called Data Link Layer (DLL) to control traffic for regulation. DLL is divided into two sub layers they are LLC-Logical link control and MAC-medium access control. MAC is a Lower part of DLL and it is responsible for Access control while the channel is accessed by multiple users simultaneously [10]. CSMA and 802.11 MAC protocols used in this paper. The brief description of these MAC protocols is described in the following subsection.

\subsection{CSMA \& 802.11}

In CSMA (Carrier Sense Multiple Access) protocol, a station wishes to transmit, first it sense (listen) the channel for to check whether it is free or not. If it is free the station can access the channel, otherwise it will wait for a random amount of time then again sense the channel [4]. Unfortunately, CSMA is restricted by two interference mechanisms: the hidden and the exposed terminal problems. In general, Due to hidden terminal collisions will occur and due to exposed terminals unnecessary delay will occur. Numerous attempts have been made to reduce the problem of hidden and exposed terminal problem. One of the mechanisms is the use of RTS/CTS (Request to Send / Clear to send) [12].

IEEE 802.11 uses RTS/CTS mechanism to reduce the problem of hidden and exposed terminal problem 802.11 differ in its requirement of an acknowledgment (ACK) transmission by the receiver after the successful reception of the data packet [9]. 802.11 define two MAC protocols, i.e., Point Coordination Function (PCF) and Distributed Coordination Function (DCF), only DCF is used in MANETs since PCF requires base stations.

\section{SIMULATION ENVIRONMENT}

This paper investigates the impact of AODV and LAR routing protocols under MAC protocols CSMA and 802.11. The simulation has been performed using the Global Mobile Information System Simulator (GloMoSim) which provides scalable simulation environment for wireless network systems [8]. The simulation area is $1000 \times 1000 \mathrm{~m}$ square topology. The node density (number of mobile nodes) is 20,40,60,80 and 100 . The node aggregation technique is used to give significant benefits to the simulation performance.

Traffic sources are Constant Bit Rate (CBR). Mobility model is Random Waypoint. Packet size is 512 bytes data. Simulation time is $300 \mathrm{sec}$. Routing protocols AODV and LAR are tested under the MAC protocols 802.11 and CSMA.

Table 1. Simulation Parameters

\begin{tabular}{|l|l|}
\hline Routing Protocols & AODV,LAR \\
\hline Simulation Time & $300 \mathrm{~s}$ \\
\hline Area (sq.m) & $1000 \mathrm{mx} 1000 \mathrm{~m}$ \\
\hline Propagation Model & Two Ray \\
\hline Traffic & CBR \\
\hline Packet Size & 512 bytes \\
\hline Nodes & $20,40,60,80,100$ \\
\hline Antenna Type & Omni directional \\
\hline Transmission range & $250 \mathrm{~m}$ \\
\hline Receiver range & $250 \mathrm{~m}$ \\
\hline Pause time & $0 \mathrm{sec}$ \\
\hline Minimum speed & $1 \mathrm{~m} / \mathrm{s}$ \\
\hline Maximum speed & $10 \mathrm{~m} / \mathrm{s}$ \\
\hline Mobility Model & Random Waypoint \\
\hline MAC & 802.11, CSMA \\
\hline
\end{tabular}

\subsection{GloMoSim}

GloMoSim is a scalable simulation environment for wireless network systems. It is being designed using the parallel discrete-event simulation capability provided by PARSEC [8]. GloMoSim simulates networks with up to thousand nodes linked by a heterogeneous communications capability that includes multicast, asymmetric communications using direct satellite broadcasts, multi-hop wireless communications using ad-hoc networking, and traditional Internet protocols. The major advantages of GloMoSim are it is globally accepted for wireless simulations. Other advantages are Achievement of large scalability, good mobility models specify for wireless simulation, and support of many ad hoc networking protocols. GloMoSim models currently available under MAC layer are 
CSMA, MACA, TSMA, 802.11. This paper examines CSMA and 802.11 for AODV and LAR routing protocols.

\section{SIMULATION RESULTS AND DISCUSSION}

The simulations were performed using GloMoSim, which provides a scalable simulation environment for wireless network systems. Number of simulation scenarios was carried out by using simulation metrics like Throughput, End-to-End Delay and Packer Delivery Ratio. Simulation results showed that LAR outperforms AODV. AODV is suitable for both 802.11 \& CSMA protocols of MAC, where as LAR is only suitable for 802.11 .

\subsection{Results of throughput}

Throughput is the average rate of successful packets delivered over a communication channel. Figures 1 \& 2 display Throughput results for AODV and LAR in 802.11 and CSMA. The experimental results states that in 802.11, LAR gives higher throughput than AODV in varying network sizes. i.e. for example when the network contains $60 \& 80$ nodes the throughput of AODV is $4083 \mathrm{bits} / \mathrm{sec}$ where as for the same network size LAR gives 4110 bits/Sec. In CSMA AODV performs well. LAR shows lot many up \& downs in throughput.

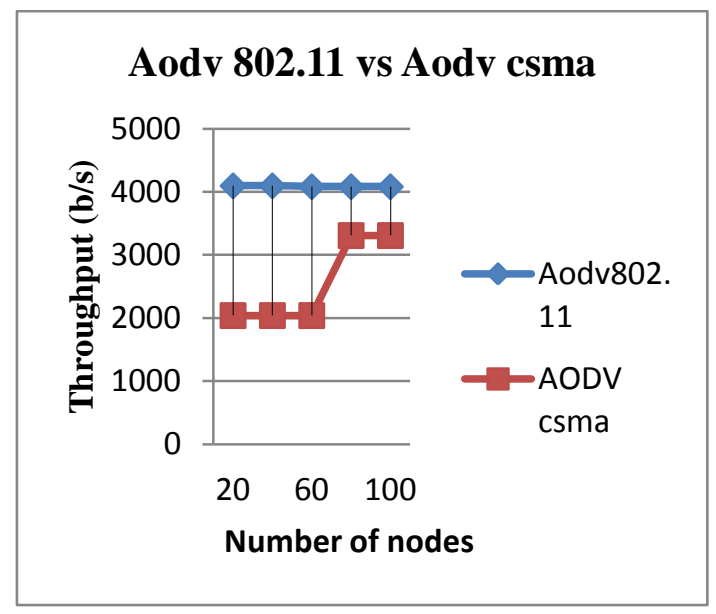

Figure 1: Variation of throughput for AODV with network size under 802.11 and CSMA

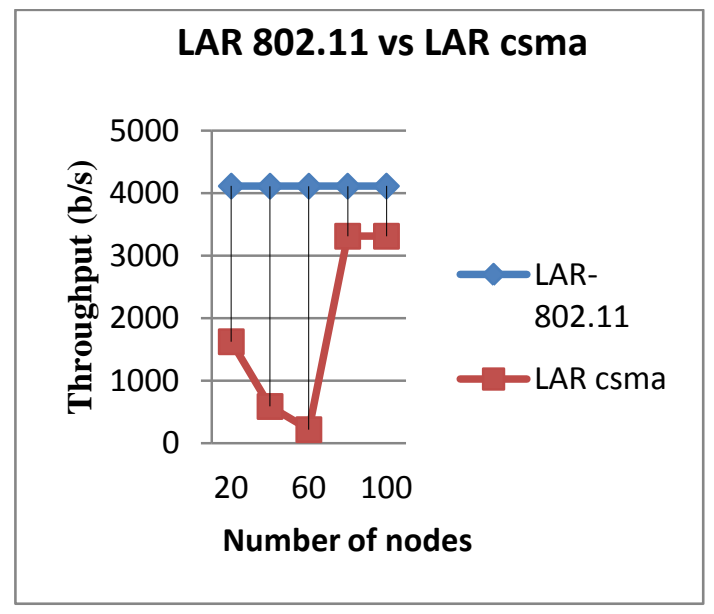

Figure 2: Variation of throughput for LAR with netwok size under 802.11 and CSMA

\subsection{Results of average end-to-end delay}

Average End-to-End Delay can be defined as a function of the signals travel time between the sender and the receiver. Figures $3 \& 4$ display the Average End-to-End delay graphs for both AODV and LAR. End-to-End delay is less for LAR in CSMA when comparing with AODV, for example if the network contains 20 nodes the delay for LAR under CSMA is $0.004371 \mathrm{~ms}$, but for same scenario delay for AODV is $0.004509 \mathrm{~ms}$, so LAR is better in CSAM. In 802.11 delay is low for LAR upto the network contains 60 nodes. If the network size increses from 60 to 100 AODV shows low delay than LAR.

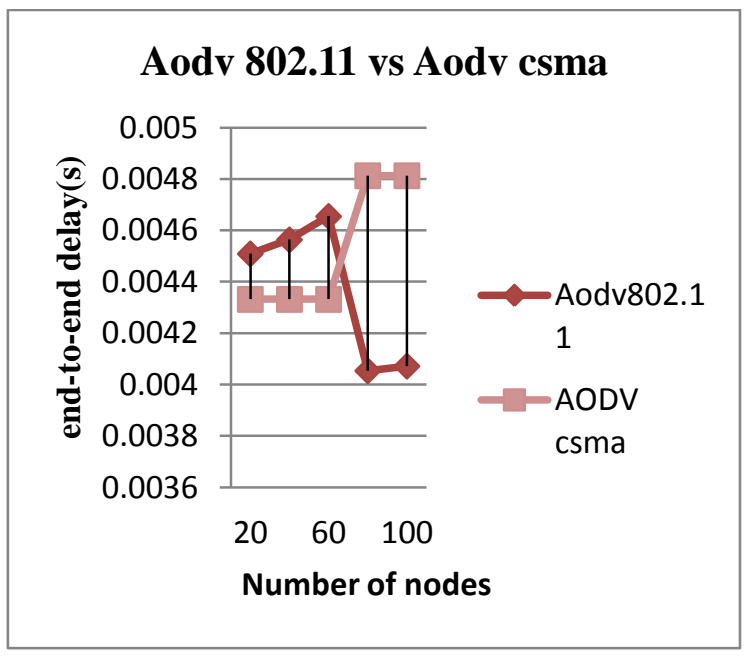

Figure 3: Variation of End-to-End Delay for AODV with netwok size under 802.11 and CSMA

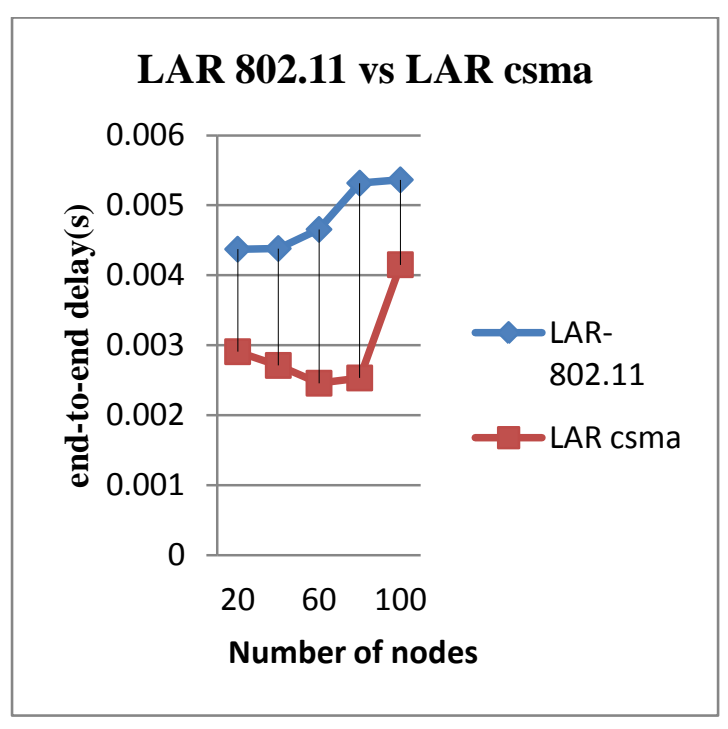

Figure 4: Variation of End-to-End Delay for LAR with netwok size under 802.11 and CSMA

\subsection{Results of packet delivery ratio}

Figures $5 \& 6$ shows the results of Packer Delivery Ratio for AODV \& LAR. Both LAR and AODV has maxmum PDR in 802.11, whereas under CSMA AODV performs better than LAR. Figure 6 specify that Packer Delivery Ratio for LAR is showing frequent up and down performances in CSMA i.e, When network contains 60 nodes PDR for AODV is $49.49 \%$ where as for LAR it is only $5 \%$. 


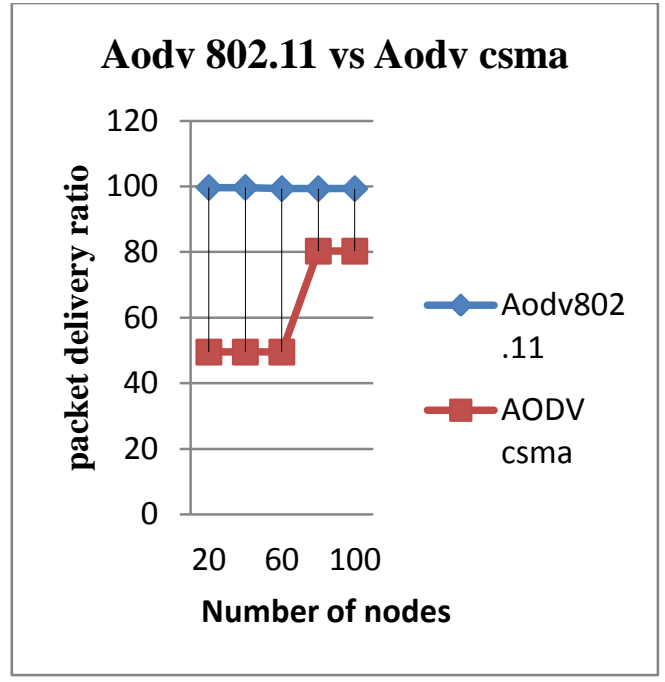

Figure 5: Variation of PDR for AODV with netwok size under 802.11 and CSMA

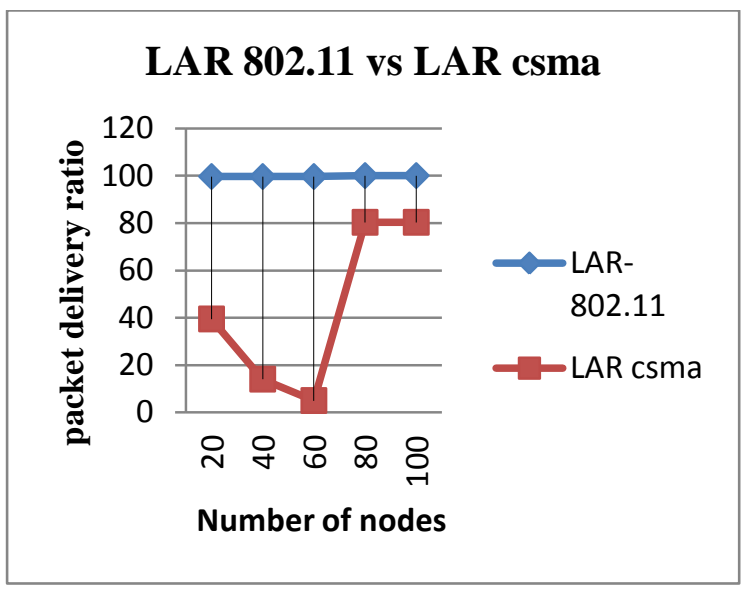

Figure 6: Variation of PDR for LAR with netwok size under 802.11 and CSMA

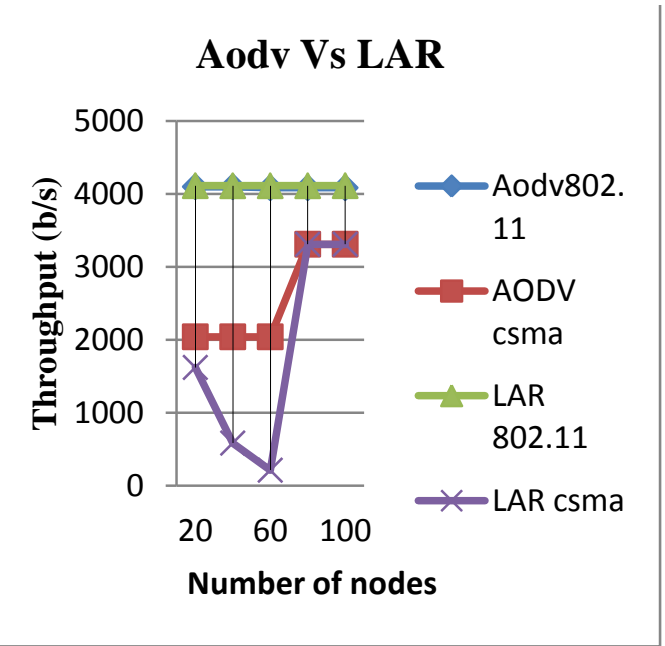

Figure 7: Variation of throughput for AODV \& LAR with network size under 802.11 and CSMA

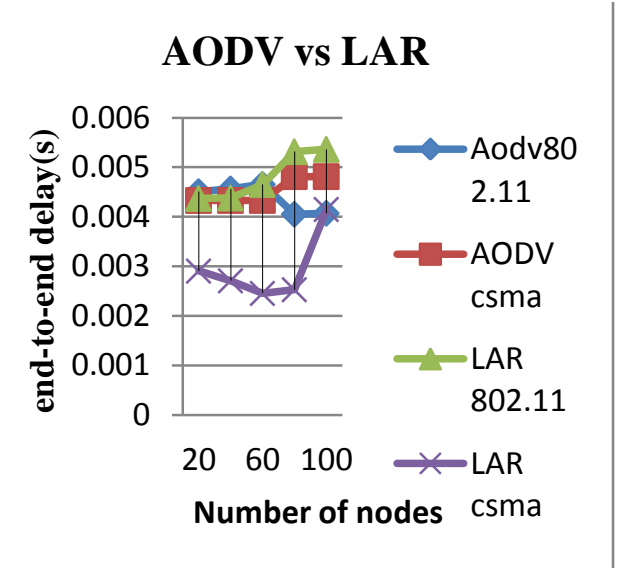

Figure 8: Variation of End-to-End Delay for AODV \& LAR with netwok size under 802.11 and CSMA

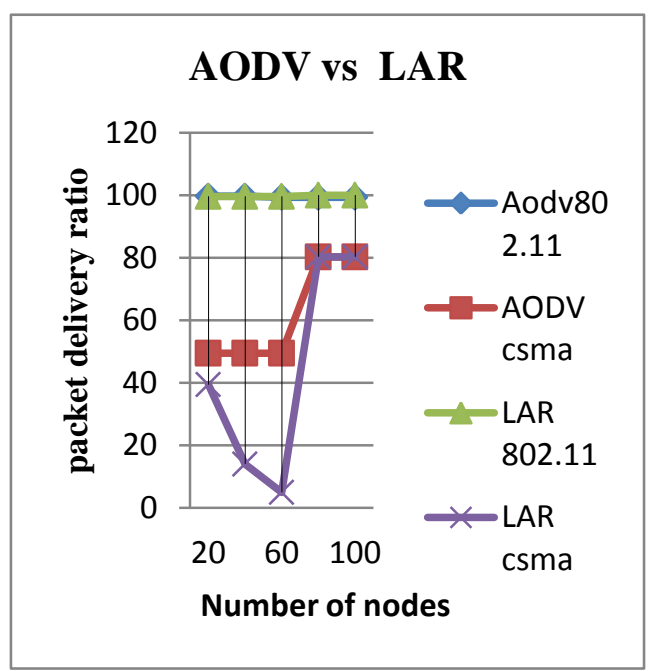

Figure 9: Variation of Packet delivery ratio for AODV \& LAR with netwok size under 802.11 and CSMA.

\section{CONCLUSIONS}

This paper examines the impact of MAC protocols 802.11 and CSMA on AODV and LAR routing protocols in MANETs. The experimental results indicate that LAR outperforms AODV in the two routing protocols under 802.11 when compared with CSMA. In this experiment uses various simulation parameters like Throughput, Average End-to-End delay and Packet Delivery Ratio. LAR is showing good throughout in 802.11 and AODV shows better throughput in CSMA. Average End-to-End delay also less for LAR in CSMA. Both AODV and LAR are showing maximum PDR in 802.11. The experiment also found that frequent up down performances in PDR under CSMA. Further work is required to compare the LAR with other protocols like ODMRP, FISHEYE under other MAC protocols like 802.11, CSMA and MACA.

\section{REFERENCES}

[1] Comparative Study of Energy Aware QoS for Proactive and Reactive Routing Protocols for Mobile Ad-hoc Networks, Dr. S.P Setty \& B.Prasad; Professor in CS \& SE, Andhra University, Visakhapatnam. International Journal of Computer Applications (0975 - 8887) Volume 31-No.5, October 2011. 
[2] Presented a paper titled "Comparative study of Reactive Routing protocols for MANETS under different traffic models in the context of Two ray and Free space Propagation Models" by Dr.S.P.Setty and A.Venkataramana; IEEE Sponsored National conference at AU-Visakhapatnam on $17^{\text {th }}$ march-2012.

[3] Impact of MAC Layer on the Performance of Routing Protocols in Mobile Ad hoc Networks, T.G. Basavaraju, Subir Kumar Sarkar and C Puttamadappa, International Journal of Information Technology Volume 3 Number 4.

[4] Performance Evaluation of Routing Protocols for MAC Layer Models, Somya Jain, Deepak Aggarwal; IOSR Journal of Computer Engineering (IOSR-JCE) e-ISSN: 2278-0661, p- ISSN: 2278-8727Volume 10, Issue 4 (Mar. - Apr. 2013), PP 71-77 www.iosrjournals.org.

[5] Energy Efficient Location Aided Routing Protocol for Wireless MANETs, Mohammad A. Mikki Computer Engineering Department IUG Gaza, Palestine, (IJCSIS) International Journal of Computer Science and Information Security Vol. 4, No. 1 \& 2, 2009.

[6] E. M. Royer and C. E. Perkins. "An Implementation Study of the AODV Routing Protocol," Proc. of the IEEE Wireless Communications and Networking Conference, Chicago, IL, September 2000.

[7] Location-Aided Routing (LAR) in Mobile Ad Hoc Networks, Young-Bae Ko and Nitin H. Vaidya Department of Computer Science Texas A\&M University.

[8] A Comprehensible GloMoSim Tutorial, compilation by Jorge Nuevo, INRS - University du Quebec nuevo@inrs-telecom.uquebec.ca, March-2004

[9] Study of different CSMA/CA IEEE 802.11-based Implementations; Authors: Miquel Oliver, Ana Escudero
Mobile Communications Research GroupDept. Mat.Apl. i Telemàtica Universitat Politècnica de Cataluña (UPC); EUNICE 1999 Contribution.

[10] The Effects of MAC Protocols on Ad hoc Network Communication Elizabeth M. Royer Sung- Ju Lee_, and Charles E. Perkins.

[11] Text Book on "Mobile Computing", Second Edition by Raj Kamal; OXFORD University Press, 2012.

[12] Text Book on "Mobile Communications", by Jochen Schiller, Addison-Wesley, second edition, 2004.

\section{AUTHORS PROFILE}

Mr. Venkataramana Attada is a research scholar in the Department of Computer Science and Systems Engineering of Andhra University, under the supervision of Professor S.Pallam Shetty. He received his M.Tech (CST) from Andhra University and presently working as Associate Professor in IT Department of GMR Institute of Technology. He is a Life Member of CSI and ISTE. He has presented more than 10 papers in various national and International Conferences. His research areas include Computer Networks, Mobile Computing, etc.

Dr. S.Pallam Shetty is a Professor in the Department of Computer Science and Systems Engineering of AU College of Engineering, Andhra University. He has guided 3 Ph.Ds and right now he is guiding $18 \mathrm{Ph} . \mathrm{D}$ scholars. He has guided more than 100 M.Tech projects. He received many honors, received Gold Medal for securing first rank in M.Sc. He has been the Resource person for various organizations. He visited Berkley University, U.S.A. He is a Life Member of IETE. He is the Editorial Board Member of various journals like IJCA, IJCIIS, and IJSAT etc. 